\title{
LA CONSTITUCIÓN DE SUBJETIVIDADES MATEMÁTICAS INFANTILES EN EL PROYECTO PEDAGÓGICO “TESELACIONES PARA NIÑOS”
}

\author{
THE CONSTITUTION OF INFANT MATHEMATICS SUBJECTIVITIES IN THE PROJECT “TESSE- \\ LLATIONS FOR CHILDREN" \\ A CONSTITUIÇÃO DE SUBJETIVIDADES DE MATEMÁTICA INFANTIL NO PROJETO “PAVI- \\ MENTAÇÕES PARA CRIANÇAS”
}

\section{Óscar Leonardo Cárdenas Forero', Sonia Milena Uribe Garzón ${ }^{2}$}

${ }^{1}$ Universidad del Tolima y Colegio Entre Nubes S.O., Bogotá, Colombia, entrenubes301@gmail.com ${ }^{2}$ Colegio Entre Nubes S. O. Bogotá, Colombia, smuribeg@gmail.com

Fecha de recepción: 12 marzo 2021

Fecha de aceptación: 25 mayo 2021

\section{RESUMEN}

Este estudio cualitativo, inscrito en el enfoque arqueo-genealógico y la analítica de la gubernamentalidad foucaultiana, es una exploración documental que busca determinar los modos como se constituyen los niños en subjetividades matemáticas, en el marco de los enunciados expresados en los documentos que hacen parte del archivo del proyecto pedagógico escolar “Teselaciones para Niños”, una alternativa didáctica en la escuela primaria para el desarrollo del pensamiento espacial y geométrico de los niños, en donde es posible visibilizar, las tecnologías de gobierno que se emplean de modo calculado, para lograr este proceso de subjetivación infantil. Con esto, además, se procura entrever la condición de mecanismos de poder que adoptan los proyectos pedagógicos que se incorporan en el aula para mejorar los desarrollos, la construcción de conocimientos y los procesos de aprendizaje infantiles. Entre los hallazgos observados, se pudieron establecer aquellos mecanismos de control que se introducen y emplean a través de las enunciaciones del proyecto, para encauzar los comportamientos de los niños y, así, conducirlos hacia la constitución de subjetividades infantiles matemáticas, que funciona conformes con los requerimientos e intereses de la racionalidad contemporánea. En conclusión, con este tipo particular de estudios, se propone analizar, cuestionar y problematizar el carácter que asumen los proyectos pedagógicos como lugares de enunciación y factibilidad de los modos de subjetivación infantil, en particular, de subjetividades infantiles matemáticas.

Palabras Claves: Tecnología de control; subjetividad; infancia; matemáticas.

\section{ABSTRACT}

This article, inscribed in the archaeo-genealogical approach and the analytics of Foucauldian governmentality, is a documentary exploration that seeks to determine the ways in which children are constituted in mathematical subjectivities, within the framework of the statements expressed in the documents that are part from the school pedagogical project "Tessellations for Children", a didactic alternative in primary school for the development of spatial and geometric thinking of children, where it is possible to make visible, the government technologies that are used in a calculated way, to achieve this process of infantile subjectivation. With this, an attempt is made to glimpse the condition of power mechanisms adopted by the pedagogical projects that are incorporated in the classroom to improve the development, the construction of knowledge and the children's learning processes. Among the find- 
ings, it was possible to establish those control mechanisms that are introduced and used through the enunciations of the project, to channel the behaviors of the children and, thus, lead them towards the constitution of mathematical infantile subjectivities, which works in accordance with the requirements and interests of contemporary rationality. In conclusion, with this particular type of studies, it is proposed to analyze, question and problematize the character assumed by pedagogical projects as places of enunciation and feasibility of the modes of infantile subjectivation, in particular, of mathematical infantile subjectivities.

Key words: Control technology; subjectivity; childhood; mathematics.

\section{RESUMO}

Este artigo, inscrito na abordagem arqueo-genealógica e na analítica da governamentalidade foucaultiana, busca determinar os modos como as crianças se constituem em subjetividades matemáticas, no quadro dos enunciados expressos nos documentos que integram a o projeto pedagógico escolar “Tesselações para Crianças”, uma alternativa didática no ensino fundamental para o desenvolvimento do pensamento espacial e geométrico das crianças, onde é possível tornar visíveis, as tecnologias governamentais que são utilizadas de forma calculada, para a concretização desse processo de subjetivação infantil. Com isso, busca-se vislumbrar os mecanismos de poder adotados pelos projetos pedagógicos que se incorporam em sala de aula para melhorar o desenvolvimento, a construção do conhecimento e os processos de aprendizagem das crianças. Dentre os achados, foi possível estabelecer aqueles mecanismos de controle que são introduzidos e utilizados por meio dos enunciados do projeto, para canalizar os comportamentos das crianças e, assim, conduzi-las à constituição de subjetividades infantis matemáticas, que funcionam de acordo com os requisitos e interesses da racionalidade contemporânea. Concluindo, com este tipo particular de estudos, propõe-se analisar, questionar e problematizar o caráter assumido pelos projetos pedagógicos como lugares de enunciação e viabilização dos modos de subjetivação infantil, em particular, das subjetividades matemáticas infantis.

\section{Palavras chaves: Tecnologia de controle; subjetividade; infância; matemática}

\section{1.- INTRODUCCION}

En los contextos escolares, en específico de educación primaria, continuamente irrumpen, se introducen y se instauran infinidad de estrategias pedagógicas, de modo alternativo, para enfrentar aquello que se encuentra institucionalizado, orientadas a la consecución de diversos propósitos, entre ellos, el mejoramiento de los aprendizajes estudiantiles, la curricularización de nuevos contenidos escolares o a la inclusión de maneras distintas para enfocar la enseñanza, las relaciones convivenciales, la producción de conocimiento y el desarrollo del pensamiento, entre otros aspectos, que se materializan, particularmente, en proyectos pedagógicos escolares.
Al tiempo que estas acciones de intervención se introducen en el aula como opciones para lograr estos objetivos, se instituyen como tecnologías de poder dirigidas a "guiar" las conductas de quienes que se involucran en ellas, constituyéndolos de forma particular, conforme, en gran medida, con la racionalidad de la época. Estas reflexiones entre 2018 y 2019, provocaron el interés por explorar y determinar en el marco del proyecto pedagógico “Teselaciones para Niños"1, los

\footnotetext{
1 "Teselaciones para Niños" es un proyecto pedagógico de aula que nació en el 2004, formulado por los maestros Sonia Milena Uribe Garzón, James Frank Becerra Martínez y Óscar Leonardo Cárdenas Forero e implementado en instituciones de educación pública primaria, en Bogotá (Colombia), enfocado al desarrollo del pensamiento geométrico y espacial, a través de las teselaciones, una propuesta artística del pintor holandés Maurits Escher, que "en el
} 
modos de subjetivación infantil propios de las "prácticas de gobierno", allí enunciadas, en una perspectiva arqueo-genealógica y de la analítica de la gubernamentalidad foucaultiana, en la que el investigador asume el papel del arqueólogo, excavando en los expedientes del proyecto, aquellos vestigios que se instalan como verdades en relación con esas formas de subjetivación infantil; pero además, el de genealogista, develando las relaciones y los mecanismos de poder que posibilitaban que dichas formas de ser sujetos en el proyecto, se instalen como legítimas y verdaderas, y como referentes para constituir a los niños en subjetividades matemáticas infantiles.

Este ejercicio metodológico, implicó un proceso de identificación, recolección y revisión de fuentes (primarias y secundarias); de lectura y tematización; de fichaje; de establecimiento de espacios de enunciación (correlativo, colateral y complementario), encuentro de regularidades; y análisis de la información y su articulación para la redacción de documentos (hallazgos y resultados), entre otros aspectos.

Gracias a esta inmersión investigativa, fue factible develar las transformaciones ocurridas en los modos de subjetivación infantil, reflejadas en el tránsito de unas tecnologías de dominación a unas tecnologías de autogobierno y la exaltación del desarrollo intelectual, apoyado por ciertas disciplinas escolares (véase las matemáticas), como políticas de subjetivación, lo que sirvió de referente para cuestionar el presente de la infancia escolarizada, hoy un asunto de debate pedagógico, psicológico, político, social y económico.

Con esto se mostró, que los proyectos pedagógicos escolares son lugares de decibilidad y factibilidad de esas maneras de producción subjetiva, donde es factible develar "los modos de subjetivación en que el ser humano se convierte en sujeto" (Chávez, 2012: 65), y en los que se determinan sus condiciones, sus actuaciones y dominio del conocimiento matemático, hace referencia al uso repetido de polígonos u otras figuras curvas que llenan completamente una región plana, infinita, sin vacíos ni superpuestos" (Uribe, Cárdenas y Becerra, 2010: 9). sus estilos de gobierno respecto a los comportamientos que se procuran inducir, así como los intereses pedagógicos, didácticos, educativos, sociales, etc., que se conjugan, los juegos de acciones acoplados y las relaciones de poder operando articuladamente, para devenir sujetos infantiles en la escuela, que obedecen a las reglas de funcionamiento de cada tiempo.

Para profundizar en este campo, se irrumpió en el corpus documental producido internamente en el proyecto pedagógico (ponencias, artículos de revista, libros, etc.); en diversos documentos que develaran las condiciones de existencia de las subjetividades actuales, como también, en los trabajos de Michel Foucault y su caja de herramientas, entre otros autores, apropiando elementos de análisis que permitieran problematizar (desnaturalizar) las maneras de subjetivación y las diversas tecnologías de control que se establecen y definen para instituir subjetividades matemáticas, en concreto, en el proyecto pedagógico objeto de estudio; lo que da apertura a cuestionarse por las formas contemporáneas (prácticas de gobierno) como se producen y convierten a los niños en sujetos matemáticos en la escuela primaria.

\section{PODER Y SUBJETIVIDAD: LOS INICIOS DE LA REVISIÓN DOCUMENTAL}

En el ejercicio investigativo, se hizo esencial, de modo preliminar, indagar los conceptos de poder y subjetividad, con el objeto de concretar un referente analítico que permitiera adelantar la excavación del archivo documental, examinando en él, los modos de subjetivación infantil y las tecnologías de gobierno que se enunciaban y utilizaban en el proyecto pedagógico para la consecución de este propósito. En esta perspectiva, se incursionó en el texto "El Sujeto y el poder", en el que Foucault (1988) define gobernar como “[...] estructurar el posible campo de acción de los otros" (p. 15). Esto es, la forma de encauzar la conducta individual o colectiva, que se manifiesta en ejercicios del poder (prácticas de gobierno) orientados a conducir efectiva- 
mente las acciones de los otros, comprendiéndose en ello, que el sujeto es libre, y, por tanto,

[...] gobernar no significa obligar a que otros se comporten de cierta forma (y en contra de su voluntad), sino lograr que esa conducta sea vista por los gobernados mismos como buena, honorable, digna y, por encima de todo, como propia, como proveniente de su libertad. (Castro, 2015: 45).

Estas "prácticas gubernamentales", según Foucault, tienen un espacio de carácter estratégico, denominado tecnología, es decir, una manera singular cómo funcionan las prácticas dentro de un dispositivo de poder. Es "la aplicación de unos medios orientados de forma consciente por la reflexión y la experiencia para alcanzar ciertos fines" (p. 36), que se utilizan de manera histórica, racional y calculada para que los sujetos se comporten con base en el establecimiento de unos propósitos predefinidos, produciendo unas subjetividades particulares conformes con dichos objetivos.

A partir de estos conceptos foucaultianos, se estableció la existencia de las tecnologías gubernamentales, susceptibles de manifestarse en múltiples lugares, por ejemplo, los proyectos pedagógicos escolares, y definidas como

[...] un nuevo conjunto que se diferencia de las tecnologías de dominación porque no buscan simplemente determinar la conducta de los otros, sino dirigirla de un modo eficaz, ya que presuponen la capacidad de acción (libertad) de aquellas personas que deben ser gobernadas. Pero también se diferencian de las tecnologías del yo, pues, aunque los objetivos del gobierno son hechos suyos libremente por los gobernados, no son puestos por ellos mismos sino por una racionalidad exterior (Castro, 2015: 41).

En este sentido, se comprendió, además, que las tecnologías de gobierno se instauran como artilugios de poder, utilizados “[...] para intentar conducir la conducta de otros conforme a metas no fijadas (aunque consentidas) por los gobernados, o bien para conducir la propia conducta conforme metas fijadas por uno mismo" (p. 41), e instaurar modos particulares de ser sujetos, los cuales, al excavar el archivo, aparecen enunciados en los proyectos pedagógicos escolares.

\section{ANÁLISIS DE LA RACIONALIDAD CONTEM- PORÁNEA}

Asumiendo este campo epistemológico, se exploraron documentalmente, las condiciones históricas contemporáneas que se establecen en relación con la constitución de sujetos, a fin de determinar las respectivas articulaciones y regularidades que existen entre los enunciados expuestos en el proyecto pedagógico “Teselaciones para Niños" y las exigencias, requerimientos, expectativas y necesidades de la racionalidad ${ }^{2}$ actual, en concreto, respecto a la formación subjetiva infantil.

En esta perspectiva, se encontró que en esta racionalidad contemporánea las prácticas de gobierno sobre las conductas individuales dejan de aplicarse en contra de la voluntad de los sujetos y de estar orientadas a su sometimiento y disciplinamiento, para procurar la capacidad de autogobierno y de actuación autónoma e independiente, constituyendo "unos sujetos morales, autorresponsables; [...] capaces de conquistar la responsabilidad, pues sólo así podrá darse "naturalmente" la congruencia de los intereses personales y los intereses colectivos" (Castro, 2015: 155). Estableciéndose, que el "[...] objetivo no es someter por la fuerza a los desobedientes, sino conducir su conducta y neutralizar su peligrosidad mediante la implementación de dispositivos de seguridad" (Castro, 2016: 12-13), lo que implica la introducción de unas estrategias de intervención, con unos objetivos y medios orientados al gobierno de las conductas de las personas.

\footnotetext{
${ }^{2}$ En este marco, "la racionalidad opera como condición de posibilidad de la acción" (Castro, 2015: 33), es decir, como el modo de funcionamiento, organización y orden de las prácticas inscritas en dispositivos de poder.
} 
Apoyados en esto, irrumpe la necesidad de formar sujetos autónomos, participativos, autogobernados, cognitivos, con habilidades intelectuales, con capacidad de resolver problemas y tomar decisiones, dispuestos a proponer alternativas de intervención y cambio a situaciones que afectan el acontecer de las comunidades en las que se encuentran inmersos. Reflexivos, participativos, competentes y comunicativos, interesados en la innovación y en la creación tecnológica, como un requerimiento contemporáneo, asociado a la introducción de una serie de tecnologías de gobierno y regulación subjetiva que garanticen la consecución libre y voluntaria de estos propósitos.

En conjunto, se privilegia la adquisición de competencias básicas y la apropiación de aprendizajes de carácter pertinente, significativo y relevante ${ }^{3}$, como ejes orientadores de los sistemas educativos mundiales, y por supuesto, de la formación escolar de aquellos "agentes productivos para la competitividad" (Martínez, 2004: 7), el mercado neoliberal y el desempeño en las organizaciones empresariales contemporáneas, pues se comprende que "son inversiones que los sujetos hacen en sí mismos, "competencias" que luego podrán capitalizar" (Castro, 2015: 205). Esto produjo que el sentido, la función y el funcionamiento de las instituciones escolares se transfigurara, dándole apertura, entre otras cosas, a la generación de estrategias de intervención pedagógica orientadas a la consecución de estos fines, pues, en la medida, en que se introdujeran estas intenciones en la cultura escolar, sería posible impulsar la constitución

\footnotetext{
${ }^{3}$ En la "Declaración Mundial sobre Educación para Todos. Satisfacción de las Necesidades Básicas de Aprendizaje” (Unesco, 1990) se expusieron, legitimaron y validaron esas herramientas esenciales para el aprendizaje (la lectura y la escritura, expresión oral, cálculo, la solución de problemas) y los contenidos básicos del aprendizaje (conocimientos teóricos y prácticos, valores y actitudes) fundamentales para que los sujetos pudieran sobrevivir, desarrollar plenamente sus capacidades, vivir y trabajar con dignidad, participar plenamente en el desarrollo, mejorar la calidad de vida, tomar decisiones y continuar aprendiendo, y por supuesto, apoyar el proceso de constitución de nuevas y distintas subjetividades, que operaron como técnicas de gobierno transformando los sistemas educativos mundiales.
}

de una subjetividad infantil creativa, autorregulada, responsable, "empresarios de su propio yo" (Gómez y Jódar, 2003: 59), conforme con las reglas de operación de la contemporaneidad.

De este modo, los sistemas educativos mundiales y las políticas de regulación y gobierno de los niños se enfocaron hacia "la formación a través del sistema escolar de sujetos creativos, flexibles, comunicativos, empáticos y polivalentes - sujetos que parecen adecuarse bien a las reglas de juego de un capitalismo postmoderno-" (Varela, 1990: 235), lo que conllevó a que, en las escuelas, en particular, las que ofrecen educación infantil y primaria, se les asignara y atribuyera la responsabilidad de iniciar tempranamente la constitución de este tipo de subjetividades infantiles.

Esto significó un cambio en el modo de operar del poder, ya que, de introducir tecnologías de sometimiento para constituir sujetos escolares, se suscitó el tránsito a la incorporación de tecnologías de gobierno, que facultarán a los niños a gestionarse a sí mismos, asumiendo el carácter de subjetividades comunicativas, intelectuales, creativas, transformadoras, críticas y propositivas de cambios. Para esto, se acudirá a la enseñanza de saberes escolares como las matemáticas, apoyándose en discursos que promulgarán la idea de "potenciar el pensamiento matemático como un reto escolar" (Ministerio de Educación Nacional (MEN), 2006: 46), considerando que "las competencias matemáticas no se alcanzan por generación espontánea sino que requieren de ambientes de aprendizaje enriquecidos por situaciones problema significativas y comprensivas, que posibiliten avanzar a niveles de competencia mucho más altos y más complejos" (p. 49) y que a través de las matemáticas, es factible iniciar a los niños en el desarrollo cognitivo, de sus procesos de pensamiento lógico y en el razonamiento.

Como se observa, no solo las condiciones, discursos, orden y estructura de las instituciones escolares sufren un proceso de transformación sino las maneras de ser de sus "prácticas de gobierno" y la forma de ser de sus 
estrategias empleadas para la conducción de las conductas y la constitución de los niños, pues, más allá de apostarle por el sometimiento subjetivo, los mecanismos de intervención se orientaban a su autogobierno y el permitirles desenvolverse libremente.

\section{4.- TECNOLOGÍAS DE GOBIERNO INFANTIL}

Hecho este análisis documental, se enfocó la excavación de los expedientes del proyecto pedagógico, hacia la determinación de las técnicas de gobierno que se utilizaban para la constitución de los niños en subjetividades matemáticas. En relación con éstas se encontró una marcada tendencia a utilizar como "tecnología gubernamental”, la configuración de ambientes de aprendizaje en el aula, en los que, no solo se acerca a los niños al mundo de las teselaciones, sino se les induce a comprenderse "sujetos que interactúan con otros sujetos” (Díaz, 1983: 12), privilegiándose una relación distinta con los demás y el conocimiento; exaltando el valor de la confianza en sí mismos, en sus criterios, intereses y habilidades; destacando la importancia del desarrollo de sus potenciales intelectuales, de la comunicación efectiva y la participación efectiva, entre otros aspectos, como condiciones para alcanzar mejores niveles de desempeño escolar y social.

En estos escenarios de aprendizaje, se promociona una serie de condiciones que incitan a que los niños aprendan a regularse a sí mismos y a autogobernarse, a estar con otros en constante actitud de aprendizaje, aprendiendo a aprender, conforme con las actividades didácticas formuladas; lo que los configura en sujetos autónomos, creativos, innovadores, emprendedores, "empresarios de sí mismos" (Castro, 2015: 175), con capacidad para actuar sobre sus aprendizajes y la producción de conocimiento, en particular, el de carácter matemático y geométrico, a quienes se les guían

[...] sus comportamientos, actitudes y valores desde principios basados en el reconocimiento del otro y de su trabajo, en la autonomía, la participa- ción, colaboración, responsabilidad y respeto por las opiniones y puntos de vista diversos, al tiempo que en el desarrollo y vivencia de capacidades como la autorregulación, gestión y toma de decisiones; todo en conjunción para reducir los niveles jerárquicos (Cárdenas, Benítez y Uribe, 2019: 41).

Esto significa la disposición de un ambiente de aprendizaje con carácter matemático en el que los niños, mediante el trabajo con las teselaciones, son influenciados a desarrollar sus habilidades espaciales (coordinación viso-motriz, coordinación figura-fondo, constancia perceptual, percepción de posición en el espacio, relaciones de percepción espacial, discriminación visual, memoria visual), a construir de manera autónoma, conocimientos, nociones y conceptos geométricos, de carácter euclidiano (línea, vértices, polígonos, etc.); topológico (región, interior, frontera); proyectivo e isometrías propias del plano euclidiano y de la geometría de las transformaciones (rotación, traslación, reflexión), así como a promover procesos de razonamiento geométrico (reconocimiento, análisis, clasificación) y artístico (apreciación estética, comunicación, creatividad, sensibilización, percepción de relaciones, atención al detalle, imaginación, entre otras), gracias a los cuales, se produce un sujeto-niño-escolar, que aprende a apropiarse, conectarse y desenvolverse tanto en el espacio representado como en el real.

Dicho ambiente de aprendizaje geométrico y espacial se configura en un "milieu", es decir, en un espacio regulado e intervenido, en el que se apuesta por la formación de subjetividades infantiles flexibles, comunicativas e intelectuales, que se apropian del espacio y la geometría a través del mundo de las teselaciones, en el que se regula la voluntad, las actitudes, los comportamientos y el modo de relacionarse, razonar y comprender de los niños. Así, se impulsa la producción de una subjetividad matemática a la que se le orientan sus comportamientos, sus modos de aprender y relacionarse con la geometría, que comprende que las matemáticas escolares son un asunto que trasciende el 
manejo de las cuatro operaciones, e incluye el campo de lo espacial y geométrico, en los que es susceptible su desenvolvimiento y desempeño eficaz, sin que sea necesaria, la existencia previa de una estructura mental o contenido preestablecido.

Para que estas condiciones de aprendizaje sean efectivas y favorables con el propósito de constituir subjetividades matemáticas, autónomas, reflexivas, comunicativas, participativas y con capacidad de trabajar con los otros, se diseñó un conjunto de unidades didácticas, conformadas por actividades de enseñanza, enfocadas al desarrollo del pensamiento geométrico y espacial, mediante la apropiación de contenidos relacionados con las teselaciones ${ }^{4}$, con las que se procuró la producción de un sujeto-niño con pensamiento geométrico y con facultad para resolver problemas espaciales. Con estas unidades didácticas: "Iniciación y exploración del mundo de las teselaciones; Introducción a las teselaciones; Características de las teselaciones; Las teselaciones y sus otras tipologías y Del mundo de las teselaciones con polígonos a los poliedros" (Uribe, Cárdenas y Becerra, 2014: 22), se privilegió el trabajo colectivo, las relaciones convivenciales armónicas, la resolución conjunta de situaciones geométricas, la colaboración y la producción colegiada del conocimiento, se condujo a los niños a emplear "[...] un vocabulario elemental en relación con las teselaciones, identifica cuadrados, rombos, rectángulos, triángulos, etc. en las teselaciones" (p. 22), a describir las características de los polígonos que constituyen las teselaciones, colorear teselaciones regulares y poligonales utilizando la Teoría de los Cuatro Colores, como también, a clasificar, dibujar $\mathrm{y}$ construir teselaciones.

Es por ello que, este modo racional de proceder del proyecto pedagógico, opera acoplado con las reglas de funcionamiento de la época actual, apostándole por la producción de sujetos-niños intelectuales, cognitivos,

${ }^{4}$ Una teselación es una composición con losetas, polígonos u otras figuras que, por repetición, cubren completamente un plano sin vacíos ni superpuestos. creadores de saber geométrico, protagonistas de su formación matemática y actores de su aprendizaje espacial, validando con ello, que "los proyectos y prácticas educativas de cada época histórica están íntimamente ligados a las formas que adopta el sistema social" (Varela, 1988: 247).

Así pues, otro de los medios calculados y racionales que se utilizaron en el marco del proyecto pedagógico, para la constitución de subjetividades infantiles matemáticas, se relacionó con la enseñanza de saberes escolares propios de las teselaciones, los cuales se curricularizaron e introdujeron como un nuevo saber escolar, que se asoció a las teselaciones poligonales, regulares, semirregulares, y teselaciones poligonales no regulares, para apoyar la gestación de ese sujeto-niño que se insinuaba, al que, según Benítez y Cárdenas (2008), se le debía desarrollar "la facultad de reconocer y discriminar estímulos visuales y de interpretarlos asociándolos con experiencias anteriores" (Frostig, 1978: 7), y sus "[...] habilidades de relaciones espaciales para organizar, analizar y sistematizar los conocimientos espaciales" (Sánchez y Bonilla, 1999: 10), pues ello, era garantía para el mejoramiento de su ubicación, orientación, localización y desempeño espacial.

En esta medida, con la enseñanza de los contenidos escolares de las unidades didácticas, se promovió una subjetividad infantil distinta (activa y dispuesta a involucrarse en su formación), y se impulsó la disolución de un sujeto-niño establecido en la escuela primaria. Es decir, aquel que para aprender matemáticas debía poseer unas estructuras mentales particulares y definidas, a quien se le ajustaban los contenidos de enseñanza, para que fuesen consonantes con sus niveles de desarrollo. Aquello que podía aprender, estaba relacionado, esencialmente, con lo aritmético y el manejo de las operaciones básicas, considerándose saberes fundamentales para su éxito escolar y su desempeño posterior en la vida social.

Esta subjetividad infantil a la que se le inculcó a pensar geométricamente y a la que se le reconocieron múlti- 
ples nociones sobre el espacio al ingresar a la escuela, "[...] uno de los aspectos que tal vez poca o ninguna tiene en la estructuración, procesos y actividades del quehacer matemático en el aula" (Cárdenas, 2004: 63), se insinuó e instaló con fortaleza mediante las tecnologías de gobierno formuladas en el proyecto pedagógico, desdibujando la formación de un sujeto-niño disciplinado, evaluado y dominado a través de los contenidos aritméticos, la mecanización de algoritmos y la apropiación de las operaciones matemáticas básicas. Al final, con las "tecnologías de gobierno" que se incorporaron a través de la propuesta pedagógica, se buscó no solo resquebrajar la condición de sujeto-niño que aprende mecánicamente y memoriza conceptos matemáticos sino contribuir en la constitución de una nueva subjetividad-niño que razona (de modo geométrico y espacial), susceptible de enfrentarse a situaciones de carácter espacial y geométrico, que identifica “[...] teselaciones en imágenes, fotografías y cuadros de arte" (Uribe, Cárdenas y Becerra, 2014: 22) y dibuja "teselaciones con plantillas, reconociendo ciertos componentes y características” (p. 22), y va más allá de la concepción aritmética para construir conocimiento matemático.

\section{5.- SUBJETIVIDADES QUE SE CONSTITUYEN}

En cuanto a los modos de subjetivación infantil, a través de las "tecnologías de gobierno" introducidas mediante el proyecto pedagógico, se impulsó la conformación de un sujeto-niño que piensa espacialmente y que resuelve problemas geométricos, en la medida en que se potencia el desarrollo de sus habilidades visuales y la construcción autónoma de conceptos geométricos, entrando en conflicto con aquella concepción establecida en la escuela, que reconoce la importancia de la apropiación de los contenidos de carácter aritmético y la mecanización de algoritmos, como manera de relacionarse y acceder a las matemáticas. Se desdibuja la imagen del sujeto-niño pasivo, receptor, que memoriza y que reproduce el conocimiento adquirido, instalado durante mucho tiempo en la escuela, para darle paso a una subjetividad que actúa libremente, que razona matemáticamente, que se relaciona geométricamente con el espacio y sus problemas, aprendiendo a ubicarse y a orientarse en él, pero, además, a dibujar, a colorear, a crear y a contrastar tonos, al momento de construir mosaicos basados en teselados.

Desde luego, las "prácticas de gobierno" formuladas por el proyecto pedagógico, se orientan a la transformación del sujeto-niño instaurado, posibilitando las condiciones para que emerja, se legitime y se acepte una subjetividad a la que se le induce a ser independiente, activa, autónoma, autorregulada, responsable de las decisiones que toma respecto a su aprendizaje $y$, que asume sus actos con compromiso, trabajando a su ritmo y construyendo conocimiento matemático con los demás.

Es por esto, que la apuesta del proyecto se concentra en desvirtuar y desvalorizar "[...] el sujeto dócil y disciplinado en virtud de la exigencia de un sujeto emprendedor, flexible, empresario de sí mismo, en permanente formación y autocreación" (Jódar, 2007: 176). En resquebrajar una subjetividad instalada, aquella que se considera no está capacitada para razonar abstractamente antes de un tiempo específico, como lo señalan Becerra y Cárdenas (2004, citando a Gattegno, 1964), o por no estar instalada "[...] en un estadio de desarrollo dado" (Gattegno, 1964: 7), privilegiando la formación de un sujeto-niño que piensa matemáticamente y que está en capacidad de comunicarse, "interpretar, predecir, conjeturar, diseñar, representar y soñar con la posibilidad de crear mundos distintos" (MEN, 2006: 46), reconociendo que sus actos de pensamiento no estaban asociados a una etapa del desarrollo determinado sino que por el contrario, “[...] [poseía] un pensamiento multivalente de gran intelectualidad y simbolismo" ( $p$. 7).

En definitiva, las “tecnologías de gobierno" se encauzan a la conformación de un sujeto-niño al que se le permite cuestionar, conjeturar, analizar y reflexionar, 
reconociéndolo como una entidad individual y diferente, que puede aprender conceptos y procedimientos matemáticos (Becerra y Cárdenas, 2004: 3-4), transformando aquella verdad, aún vigente en la escuela, de que los niños deben atravesar ciertas etapas que van de lo concreto a lo gráfico y desde éste a lo abstracto para aprender y apropiarse de los conceptos matemáticos, lo que ha desembocado en la permanencia de la confusión de que los aprendizajes espaciales ligados a la matemática son aquellos que están acoplados al movimiento o a los desplazamientos que se realizan sobre el espacio (Broitman, 2000). Es por este motivo, que se les permite a los sujetos-niños enfrentarse a la construcción de

[...] conocimientos relativos a la orientación y localización en el espacio, la representación de posiciones y desplazamientos propios y de los objetos con la construcción de sistemas de referencias. Esto implica la producción e interpretación de representaciones gráficas del plano y los conocimientos vinculados a los cambios de puntos de vista [...] [y a la producción de] conocimientos relativos a las figuras geométricas y cuerpos. La exploración y el análisis de formas geométricas, la observación y la descripción de sus características a partir de las relaciones entre unas y otras, la reproducción, la representación y la construcción de figuras (Quaranta y Ressia, 2009: 9) ${ }^{5}$

Así, la configuración de un sujeto-niño distinto se legitima a través de las "prácticas de gobierno" formuladas e implementadas por el proyecto pedagógico, "inventando" una subjetividad competente matemáticamente, que piensa de manera abstracta y que aprende que

\footnotetext{
5"El primero de estos dos ejes hace referencia a una serie de conocimientos necesarios para el dominio de las relaciones espaciales tales como la orientación en el espacio, la ubicación de un objeto o persona, la organización de desplazamientos, la comunicación de posiciones y desplazamientos, la producción e interpretación de representaciones planas del espacio. El segundo hace referencia a las propiedades vinculadas a las formas geométricas (figuras y cuerpos)" (Quaranta y Ressia, 2009: 9).
}

las matemáticas escolares no son un saber reservado para "personas especiales o especialmente dotadas" (Escuela Pedagógica Experimental (EPE), 1989: 12). Con las apuestas del proyecto pedagógico, se trastocan las "prácticas de gobierno infantil" en el aula y la escuela, en términos de conducir las conductas de los niños, hacia la configuración de una subjetividad infantil que piensa espacial y geométricamente, que "[...] llega a la escuela conociendo algo de su propio mundo espacial, pero conociendo poco de matemática" (Feria, Espinosa y Martínez, 2006:19), cuyo aprendizaje es posible en la medida en que se explore el mundo visual que le rodea, que "[...] necesita enriquecer y estructurar las experiencias espaciales de los alumnos, desarrollar su vocabulario relativo al espacio y crear las condiciones para que su capacidad de visualizar el espacio se pueda explotar al máximo" (Bishop, 1986:187), que se desempeña con eficiencia al momento de solucionar problemas relacionados con la localización, orientación, ubicación, representación y aprehensión espacial.

\section{6.- REFLEXIONES FINALES}

La escasez de estudios enfocados a mostrar a los proyectos pedagógicos escolares como dispositivos en los que es posible rastrear los modos de subjetivación de los niños en la escuela, y los medios calculados y racionales que se emplean para ello, se convierte en una posibilidad para insertar estudios orientados en esta vertiente, en tanto, permite determinar, analizar, y cuestionar las prácticas de gobierno que se instalan para conducir las conductas de los sujetos escolares, y con ello, visibilizar "la relación entre las prácticas de subjetivación y juegos de verdad [...]" (Castro, 2014: 136). Con base en esto, se formuló este ejercicio investigativo, que, gracias a la inmersión en el archivo documental del proyecto pedagógico "Teselaciones para Niños", se pudieron develar los modos de subjetivación y las "tecnologías de gobierno" que se emplean para constituir de manera singular a los niños en subjetividades matemáticas. Gracias a esto, fue factible deter- 
minar y analizar las relaciones de poder que hacen de los niños en la escuela primaria, subjetos-matemáticos, que se cuestionan, relacionan con el espacio a través del desarrollo de sus habilidades espaciales y de los conceptos geométricas, conjeturan, toman decisiones y participan de la producción de conocimiento en el aula.

En esta perspectiva, no solo se visibilizaron los proyectos pedagógicos escolares como lugares de enunciación y factibilidad de esas formas de subjetivación y en los que se puede vislumbrar la manera como opera el poder sino se reconoció que los sujetos que participan de estas alternativas que intervienen en la escuela, tienen "[...] la capacidad de estar en constante elaboración" (Chávez, 2012: 81-82), los cuales se definen, "fabrican" e implementan conforme con las reglas de funcionamiento de esta racionalidad actual. Entre esas "tecnologías de gobierno", el proyecto pedagógico le apuesta por la creación de ambientes de aprendizaje en los que se propician ciertas condiciones de aceptabilidad para gobernar las conductas infantiles y orientarlas hacia el desarrollo de "[...] su capacidad de pensamiento y de reflexión lógica sino que, al mismo tiempo, [adquirir] un conjunto de instrumentos para explorar la realidad, representarla, explicarla y predecirla; en suma; para actuar en y para ella" (MEN, 1998:35), de sus habilidades visuales y geométricas, como condición de construcción, apropiación, significación, ubicación y desenvolvimiento espacial, pero también, para la configuración de sujetos-niños autónomos, singulares, intelectuales, activos, comunicativos, autorregulados y reflexivos, que razonan sin depender necesariamente de sus estadios de desarrollo, que ingresan a la escuela con múltiples nociones espaciales, que desarrollan su capacidad de trabajar en equipo y tomar decisiones colegiadas, adquiriendo aquellos desarrollos y aprendizajes que les permiten desempeñarse en el contexto escolar y desenvolverse en la vida profesional.

Con esto, se deja de privilegiar el establecimiento de un sujeto-niño pasivo, memorístico y mecánico, al que se le valida en la medida en que se desenvuelva en el campo de lo aritmético y en el manejo de algoritmos propios de las operaciones básicas, inscrito desde hace mucho tiempo en la escuela, transformando el modo como el poder funciona en la actualidad. En definitiva, excavar los terrenos en los que se cimientan los proyectos pedagógicos escolares, con la intención de visibilizar los modos de subjetivación, los juegos de verdad y las prácticas de gobierno que se utilizan para ello, se constituye en una posibilidad para develar una forma particular de funcionamiento, es decir, como tecnología de poder mediante la cual, calculada y racionalmente, se induce la manera como se deben comportar los sujetos-niños en la escuela y la sociedad.

\section{Referencias bibliográficas}

Becerra Martínez, J. F. \& Cárdenas Forero, Ó. L. (2004). Pensar matemáticamente: Una manera distinta de enfocar el ambiente matemático en la escuela (Ponencia sin publicar). Bogotá.

Benítez, M. L. \& Cárdenas, Ó. L. (2008). La enseñanza de la topología a través de la cartografía. Bogotá: Cooperativa Editorial Magisterio. https://doi. org/10.17227/01224328.1233

Cárdenas Forero, Ó. L. (julio - diciembre, 2004). La enseñanza de la Topología a través de la Cartografía Nodos y nudos, 2 (17). Universidad Pedagógica Nacional (UPN). Bogotá: Panamericana Formas e Impresos S. A. https://doi.org/10.17227/01224328.1233

Cárdenas Forero, Ó. L., Benítez Agudelo, M. L. y Uribe Garzón, S. M. (julio-diciembre, 2019). La constitución de los maestros en el marco de las redes y colectivos: el caso de la red "maestros en colectivo". Educación y Ciudad. 2 (37). pp. 37-46 IDEP - DOI: https://doi. org/10.36737/01230425.v2.n37.2019.2146

Castro, E. (2014). Introducción a Foucault. Siglo XXI Editores.

Castro- Gómez, S. (2015). Historia de la gubernamen- 
talidad I. Razón de Estado, liberalismo y neoliberalismo en Michel Foucault. Bogotá: Siglo del Hombre Editores. https://doi.org/10.15332/s0120-8462.2011.0105.07

Castro- Gómez, S. (2016). Historia de la gubernamentalidad II. Filosofía, cristianismo y sexualidad en Michel Foucault. Bogotá: Siglo del Hombre Editores.

Bishop, A. (1986) ¿Cuáles son algunos obstáculos para el aprendizaje de la geometría? Estudios en Educación Matemática. Enseñanza de la Geometría. 5. París: Editado por R. Morris. Publicado por Unesco. https://doi. org/10.12795/jdu.2018.i01.44

Broitman, C. (2000). Reflexiones en torno a la enseñanza del espacio. Educación Matemática. Propuestas de trabajo, experiencias y reflexiones. La educación en los primeros años. Buenos Aires: Ediciones Novedades Educativas. https://doi.org/10.3989/ arbor.2011.749n3005

Del Grande, John. (1987). Spatial Perception and Primary Geometry. Learning and Teaching Geometry, K-12- Yearbook of the National Council of Teachers of Mathematics, edited by Mary Montgomery. Lindquist. Reston, VA.

Diaz, E. (1993). Michel Foucault. Los modos de subjetivación. Colección Perfiles. Editorial Almagesto.

Chávez Muriel, H. R. (2012). Un acercamiento al concepto de sujetos en el pensamiento de Michel Foucault. Del ser humano al sujetos y el gobierno de sí mismo com práctica de libertad. Universidad del Valle. Programa Editorial. https://doi.org/10.2307/j.ctvt9k328.16

Escuela Pedagógica Experimental (EPE). (1989). Coloquio de Matemáticas. Bogotá.

Feria Uribe, M. A., L. B. Espinosa \& Martínez Álvarez, N. (2006). Percepción espacial y geometría intuitiva. Una puerta de entrada al aprendizaje significativo de la geometría. Bogotá: Universidad Externado de Colombia, Facultad de Ciencias de la Educación. https://doi. org/10.2307/j.ctv13vdfnj.8
Foucault (1984). La ética del cuidado de sí como práctica de la libertad. Diálogo con H. Becker, R. Fornet-Betancourt, A. Gómez-Müller, 20 de enero. (pp. 257-280) En: https://revistas.unc.edu.ar/index.php/NOMBRES/ article/viewFile/2276/1217 https://doi.org/10.2307/j. ctvb6v7nm.6

Foucault, M. (1988). El sujeto y el poder. Revista Mexicana de Sociología, Vol. 50, No. 3. (jul. - Sep.), pp. 3-20. En: http://links.jstor.org/sici?sici=01882503\%28198807\%2F09\%2950\%3A3\%3C3\%3AESYEP \%3E2.0.CO\%3B2-A https://doi.org/10.2307/3540551

Foucault, M. (2008). Tecnologías del yo y otros textos afines. Barcelona: Ediciones Paidós Ibérica, S.A.I.C.E. de la Universidad Autónoma de Barcelona.

Frostig, M. (1978). Figuras y formas. Programa para el desarrollo de la percepción visual, Buenos Aires: Médica Panamericana.

Gattegno, C. (1964). El material para la enseñanza de las matemáticas. Madrid: Aguilar Editores.

Gómez, L. \& Jódar, F. (2003). Foucault y el Análisis Sociohistórico: Sujetos, saberes e instituciones educativas. En: Foucault, la educación y la pedagogía. Revista Educación y Pedagogía. Facultad de Educación. No. 37. Vol. 15. pp. 55-68. Medellín: Universidad de Antioquia. https://doi.org/10.17227/01212494.3pys34.51

Jódar, F. (2007). Alteraciones pedagógicas. Educación y políticas de la experiencia. Barcelona: Laertes Educación.

Martínez Boom, A. (2004). De la Escuela Expansiva a la Escuela Competitiva. Barcelona: Antropos Editorial. Ministerio de Educación Nacional (MEN). (1998). Lineamientos Curriculares Matemáticas. Bogotá: Editorial Delfín Ltda.

Ministerio de Educación Nacional (MEN). (2006). Estándares Curriculares. Bogotá.

Quaranta, M. E., y B. Ressia de Moreno (2009). La enseñanza de la geometría en el jardín de infantes. (Serie 
Desarrollo Curricular). La Plata: Dirección General de Cultura y Educación de la Provincia de Buenos Aires. https://doi.org/10.35537/10915/29366

Sánchez, N., y M. Bonilla (1998), Matemáticas escolares asistidas por computador. Actividades en el aula. Módulo 3, Proyecto curricular de Licenciatura en Matemáticas, Bogotá: Universidad Distrital.

Unesco. (1990). Declaración Mundial sobre Educación para Todos. Satisfacción de las Necesidades Básicas de Aprendizaje. En: http://www.unesco.org/education/ pdf/JOMTIE_S.PDF https://doi.org/10.5944/educxx1.3.1.405

Varela, J. (1988). La Educación Ilustrada o como fabricar sujetos dóciles y útiles. Universidad Complutense. (pp.245-274). En: http://www.educacionyfp.gob.es/ dam/jcr:d86b8ec4-4c09-4957-b3d6-6c651ca19139/ re198812-pdf.pdf

Varela, J. (1990). Clases sociales, pedagogías y reforma educativa. Revista de Educación. Formación general, conocimiento escolar y reforma educativa (y II). No. 292. Mayo-agosto. Madrid: Secretaría General Técnica, Subdirección General de Atención al Ciudadano, Documentación y Publicaciones. (pp. 219236). En: https://sede.educacion.gob.es/publiventa/ descarga.action?f_codigo_agc=478_19 https://doi. org/10.35376/10324/1792

Uribe, S. Cárdenas, O. \& Becerra, J. (2010). Teselaciones para niños: una mirada genealógica a las prácticas de enseñanza de las matemáticas en la escuela (2004- 2010). Informe final. https://doi.org/10.35537/10915/75690

Uribe, S. Cárdenas, O. \& Becerra, J. (2014). Teselaciones para niños: una estrategia para el desarrollo del pensamiento geométrico y espacial de los niños. Educación Matemática, vol. 26, núm. 2. Agosto. (pp.135-160). En: http://www.scielo.org.mx/scielo.php?script=sci_arttex t\&pid=S1665-8262014000200005 